University of Wollongong

Research Online

Australian Institute for Innovative Materials -

Papers

Australian Institute for Innovative Materials

$1-1-2019$

\title{
Using force-displacement relations to obtain actuation parameters from artificial muscles
}

\section{Geoffrey M. Spinks}

University of Wollongong, gspinks@uow.edu.au

Shannon Bakarich

Cornell University, seb798@uowmail.edu.au

Shazed Md Aziz

Linkoping University, shazed@uow.edu.au

Bidita Binte Salahuddin

University of Wollongong, bbs622@uowmail.edu.au

Hai Xin

University of Wollongong, hxin@uow.edu.au

Follow this and additional works at: https://ro.uow.edu.au/aiimpapers

Part of the Engineering Commons, and the Physical Sciences and Mathematics Commons

Research Online is the open access institutional repository for the University of Wollongong. For further information contact the UOW Library: research-pubs@uow.edu.au 


\title{
Using force-displacement relations to obtain actuation parameters from artificial muscles
}

\author{
Abstract \\ Many different test methods are currently used to characterise the output of artificial muscle materials \\ but few studies report the full range of possible force and displacements that can be generated by a given \\ material when activated with a given input stimulus but when operated against different external loads. \\ The measurement of the loading and unloading force extension curves in tension in both the un-activated \\ and activated states is investigated as a means for efficiently characterising the full range of outputs for \\ three different types of artificial muscles: pneumatically operated braided muscle and thermally operated \\ shape memory alloy spring and twisted / coiled polymer fiber. A graphical method of analysis was applied \\ whereby the force-extension curves obtained before and after actuator activation were plotted on the \\ same axes. By overlaying the external loading conditions, the graphical method provided the equilibrium \\ starting and finishing forces and displacements and successfully predicted the isotonic strokes, isometric \\ forces and combined force and displacement generated when the actuator was operated against an \\ external spring. Complications in the interpretation of the force-stroke curves were encountered as all \\ three artificial muscles displayed a degree of loading-unloading hysteresis and non-ideal mechanical \\ behavior.

\section{Disciplines} \\ Engineering | Physical Sciences and Mathematics

\section{Publication Details} \\ Spinks, G. M., Bakarich, S. E., Aziz, S., Salahuddin, B. \& Xin, H. (2019). Using force-displacement relations \\ to obtain actuation parameters from artificial muscles. Sensors and Actuators, A: Physical, 290 90-96.
}




\section{Using force-displacement relations to obtain actuation parameters from artificial muscles}

Geoffrey M. Spinks ${ }^{1 *}$, Shannon E. Bakarich ${ }^{2,3}$, Shazed Aziz ${ }^{4}$, Bidita Salahuddin ${ }^{1}$ and Hai Xin ${ }^{1}$

1. AlIM Facility, University of Wollongong, Squires Way, North Wollongong, NSW, 2522, Australia.

2. U.S. Army Research Laboratory, 2800 Powder Mill Road, Adelphi, MD 20783, USA

3. Department of Mechanical and Aerospace Engineering, Cornell University, Ithaca, NY 14850, USA

4. Department of Physics, Chemistry and Biology, Linköping University, Linköping 581 83, Sweden.

\section{Introduction}

Many types of mechanical actuators, or 'artificial muscles', have been developed and considered for a myriad of applications including robotics [1] and medical devices [2]. The fundamental performance properties of these artificial muscles are the force generated and the stroke, or the extent of displacement achieved, when the material is given an input stimulus [3]. Very few studies fully characterise the actuator's output in terms of both the displacement and the force generated [4]. Some researchers simply report the deformation, especially when the actuator generates a bending or torsional response.[5] In some cases, the force generated is reported when all deformation is constrained [6], which is called the blocked force. The situation is further complicated by the fact that the actuator's output is influenced by the external loading conditions. It is often seen that the actuator's stroke changes when different pre-load forces are applied before the actuator is stimulated. $[7,8]$ Some studies have also shown that the blocked force can be influenced by the extent of pre-loading. [6,9] These effects mean that it is impossible to simply characterise an artificial muscle as one that generates a certain stroke and force (or strain and stress). What is needed is a more systematic way to determine an artificial muscle's performance under any external loading conditions. This type of information is critically important when the artificial muscles are to be used in load-bearing applications including exoskeletons [10], prosthetics [11], and robotic devices. [12]

Early work on shape memory alloy (SMA) actuators used the material's force-displacement relationships as a means for understanding the effects of external loads on the shape memory behaviour. [13] SMAs only generate an actuation force or stroke after the material has been mechanically deformed by a pre-load and then subsequently heated to generate the 'recovery'. The thermo-mechanical properties of the material, therefore, are critically important to the SMA effect. Tobushi and Tanaka [13] have used the material's force-displacement curves experimentally determined at different temperatures to illustrate the effect of mechanical deformation on the extent of the shape recovery. As described in detail below, a graphical method using forcedisplacement curves obtained before and after activation can be used to predict the free stroke, the blocked force, and the isotonic strokes and isometric forces generated at any pre-load. In addition, situations where the external loads change during the actuation cycle, such as when the actuator 
operates against a spring, can also be predicted. The method appears to be universally applicable to any actuator and it is here applied to three types of tensile actuators: a SMA spring; a pneumatically operated braided muscle; and a twisted and coiled polymer fiber. The advantage of this test method is that it is simple and quick to perform and avoids the laborious multiple testing used in previous studies. $[7,8]$

\section{Theoretical Basis for a Graphical Method of Analysis}

The three common modes of operation for tensile actuators are illustrated in Figure 1 as isotonic (constant load), isometric (constant length) and against an opposing spring (changing length and load). Plotting the force-extension curves of the actuator both before and after activation on the same axes provides a means for graphically estimating the actuation behaviour. For any loading scenario, the actuation stroke and force generated can be represented by the transition between the starting equilibrium point, which lies on the initial (un-activated) force-extension line, and the final equilibrium point, which falls on the force-extension line in the activated state. For example, the isotonic actuation is illustrated in Fig. 1a where pre-loading is represented by point A on the forceextension curve in the initial, un-activated state. Stimulating the actuator while maintaining a constant force is illustrated by the horizontal line joining the initial equilibrium point $A$ with the final equilibrium point $B$. The latter lies on the force-extension curve for the actuator in its final, activated state. The isotonic actuation stroke is determined by the horizontal distance between points A and B. The situation illustrated in Figure 1 shows an actuator that contracts in length with a decrease in stiffness when activated, with the stiffnesses represented by the slope of the force-extension curves. The graphical method readily illustrates that the isotonic actuation stroke depends on the pre-load force applied before actuator activation. In the example illustrated in Fig. 1a, the isotonic stroke decreases with increasing pre-load and the maximum stroke occurs at a zero pre-load force. This property is sometimes referred to as the 'free stroke' $\left(\Delta \mathrm{L}_{0}\right)$ and is the change in length of the actuator in the absence of any externally-applied load.

Examples of alternative loading conditions are illustrated in Figs. $1 \mathrm{~b}$ and $1 \mathrm{c}$. In each case, the per-load force is indicated by point $A$ on the initial force-extension curve. The length of a vertical line from point $A$ to point $C$ is used to graphically estimate the isometric actuation force generated by activation of the actuator (Fig. 1b). In the case illustrated for a contractile actuator that reduces stiffness when activated, the generated isometric force decreases in magnitude as the applied preload force increases. The maximum generated force in this case occurs at a zero pre-load, which is called the 'blocked force' $\left(\Delta \mathrm{F}_{\mathrm{b}}\right)$. Operating the actuator against a spring in series is analysed by drawing a force-extension curve for the external spring so that the slope of the line is equal to the external spring constant. The pre-load force due to stretching of both the spring and the actuator is again indicated by point $A$ in Fig. 1c. The force generated and stroke when the actuator operates against this spring in series is determined by the vertical and horizontal distances, respectively, from point $A$ to point $D$ (Fig. 1C). The latter corresponds to the intersection of the external spring forceextension curve and that of the actuator in the activated state.

The graphical method of analysis can be applied to any external loading condition by identifying the equilibrium starting and finishing load and displacement of the actuator. Sometimes, the mix of force and displacement generated by the actuator is summarised in a force-stroke curve (Fig. 1d). For ideal elastic materials the force stroke curve is a straight line joining the free stroke $\left(\Delta \mathrm{L}_{\mathrm{o}}\right)$ with the blocked 
force $\left(\Delta \mathrm{F}_{\mathrm{b}}\right)$. If the stiffness of the actuator material does not change on activation, then the forcestroke curves are independent of the initial pre-stretch applied to the actuator. However, for actuators that show a change in stiffness, then the force-stroke curves will be different for each applied pre-stretch. For the actuator illustrated in Figure 1 the activated state has a lower stiffness than the actuator's un-activated state. Consequently, the isotonic stroke and isometric force generated will decrease in magnitude as the pre-stretch applied to the un-activated actuator increases. In the ideal case, the full range of actuator performance can be determined from three parameters: the free stroke and the stiffness in the initial and activated states.
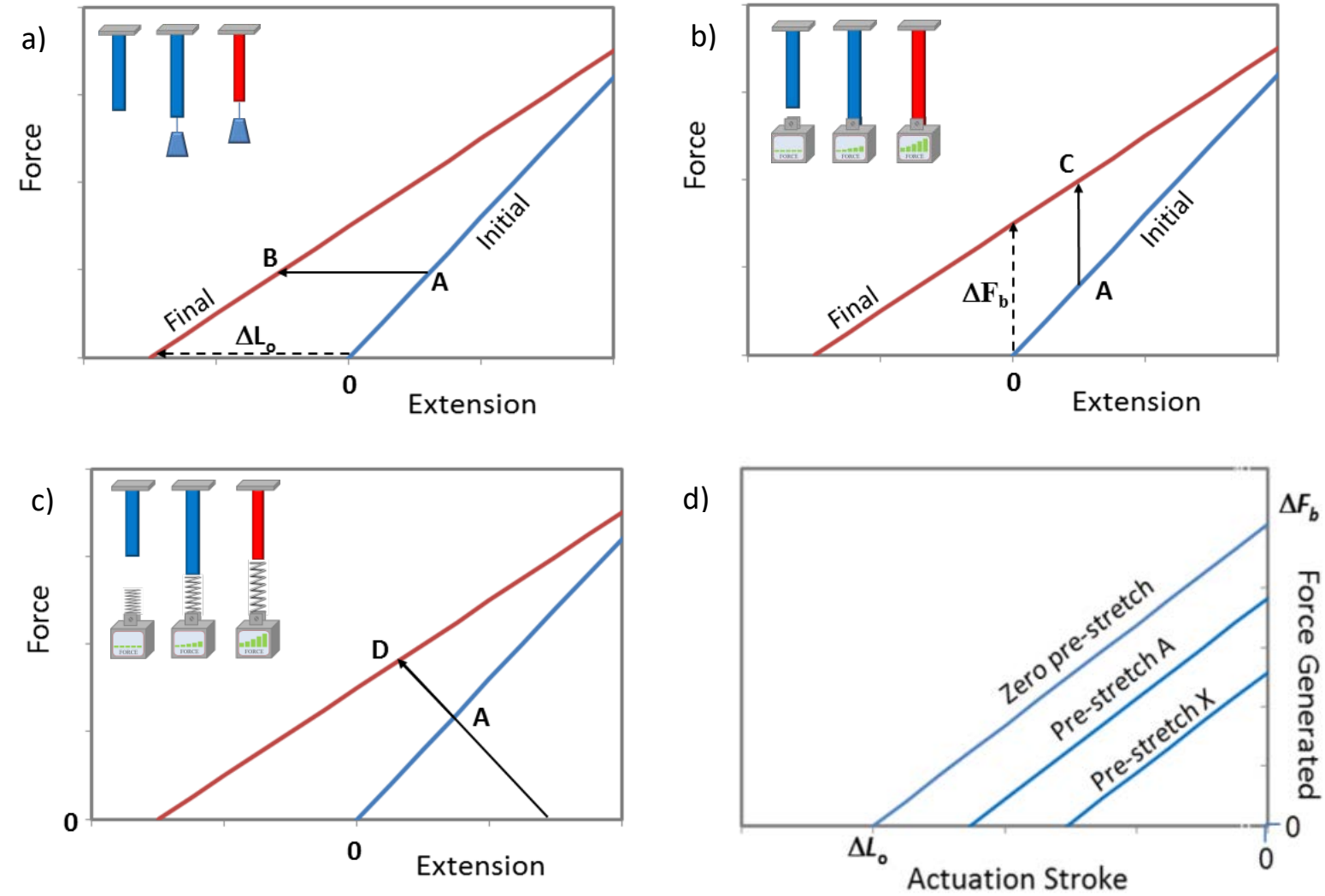

Figure 1 Illustration of the three common actuation modes: a) isotonic; b) isometric and c) against a spring. In the latter, the spring stiffness is represented by the slope of the line A-D. Force-extension curves for the actuation in the initial (unactivated) and final (activated) states are shown as straight lines in a) - c). The force-stroke curves for three different pre-stretches are illustrated in d). Inset figures in a)-c) schematically show from left to right the initial, pre-loaded and final states.

\section{Experimental}

Actuation tests were performed on three types of tensile artificial muscles that contract in length when stimulated (Fig. 2): a pneumatically operated braided muscle, a thermally operated shape memory alloy (SMA) spring, and a thermally operated twisted and coiled polymer (TCP) fiber. The braided muscle was constructed from $6 \mathrm{~mm}$ diameter polyester braid and fitted with a $5.5 \mathrm{~mm}$ diameter natural rubber internal bladder. The ends were sealed with pneumatic fittings connected to 
a pneumatic piston (syringe) at one end and a pressure sensor (Gems Sensors and Control) at the other end. The active length of the unpressurised braided muscle was $71 \mathrm{~mm}$. The SMA spring was purchased from Dynalloy Inc. (3-642 NiTi tension spring) and was fabricated from $0.75 \mathrm{~mm}$ diameter nitinol wire to give a spring of $5 \mathrm{~mm}$ diameter and $15 \mathrm{~mm}$ in length. The TCP fiber was made from 0.7 mm diameter nylon- 6 monofilament (Jarvis Walker Bulk Mono Line, $50 \mathrm{lb}$ ) that was twisted until it was fully coiled and then annealed at $180^{\circ} \mathrm{C}$ under tension for 2 hours followed by slow cooling. The final coiled fiber length was $58 \mathrm{~mm}$ and coil outer diameter was $1.8 \mathrm{~mm}$.

a)

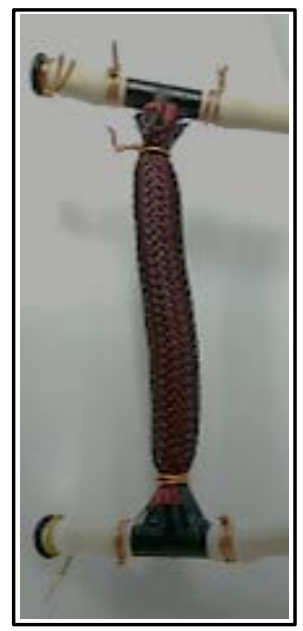

b)

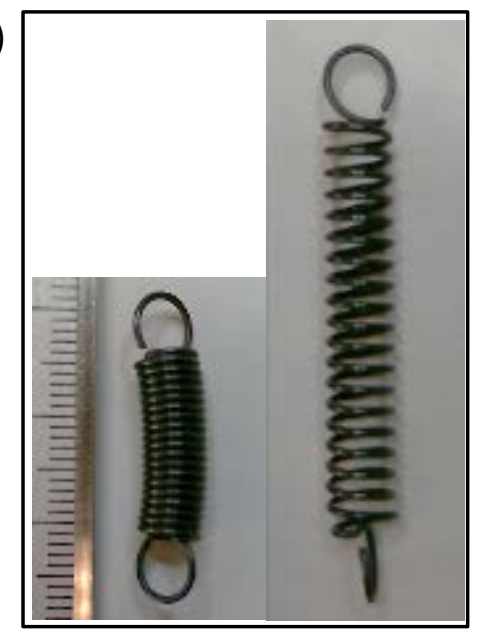

c)

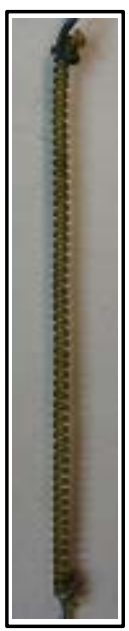

Figure 2 Photographs of the three types of contractile tensile actuators used in this study: a) braided pneumatic muscle; b) SMA spring in the fully contracted and an example pre-stretched state; c) TCP fiber.

Each type of actuator was subjected to three types of actuation tests: isotonic, isometric and when operated against a spring in series. In each test, the sample pre-load was systematically varied. Only one level of input stimulus was applied for each type of artificial muscle: $100 \mathrm{kPa}$ air pressure for the braided muscle; or circulating water at $85^{\circ} \mathrm{C}$ and $25^{\circ} \mathrm{C}$ for the SMA spring and for the TCP fiber. The actuation response was determined once the displacement and/or force change had reached equilibrium, which was approximately 60 seconds after applying the temperature or pressure stimulus. In addition, the force-extension curves for each actuator were measured in both the activated and non-activated states. These tests were performed by loading and unloading the sample at constant pressure (braided muscle) and constant temperature (SMA spring and TCP fiber). Helical steel springs with stiffnesses in the range $0.6-1.9 \mathrm{~N} / \mathrm{mm}$ were used in some actuator tests, as indicated. A custom built experimental set-up comprising a load cell and screw-driven crosshead was used to load / unload the muscles at a crosshead speed of $\sim 50 \mathrm{~mm} / \mathrm{min}$. To achieve isobaric conditions when testing the braided muscles, a series of loading and unloading increments were imposed and after each change in the sample length, the internal pressure difference was adjusted to the target value of either $0 \mathrm{kPa}$ or $100 \mathrm{kPa}$. 


\section{Results and Discussion}

The actuation test results for the braided muscle are given in Figure 3 as a function of pre-load applied in each case. The pre-strains were in the range of 0 to $5 \%$. The magnitude of the isotonic contraction diminished with increasing pre-load applied to the braided muscles (Fig. 3a). The maximum strain obtained was the free stroke of $-11 \%$, which is the change in actuator length at zero applied load and when normalised to the unloaded length. Similarly, the force generated during the isometric tests decreased with increasing pre-load (Fig. 3b). The maximum blocked stress of 0.34 MPa was calculated from the maximum force generated per unloaded cross-sectional area of the actuator. When operated against a spring in series with a spring constant of $0.8 \mathrm{~N} / \mathrm{mm}$, both the force generated and the contraction again decreased with increasing pre-load (Figs. $3 \mathrm{c}$ and d).
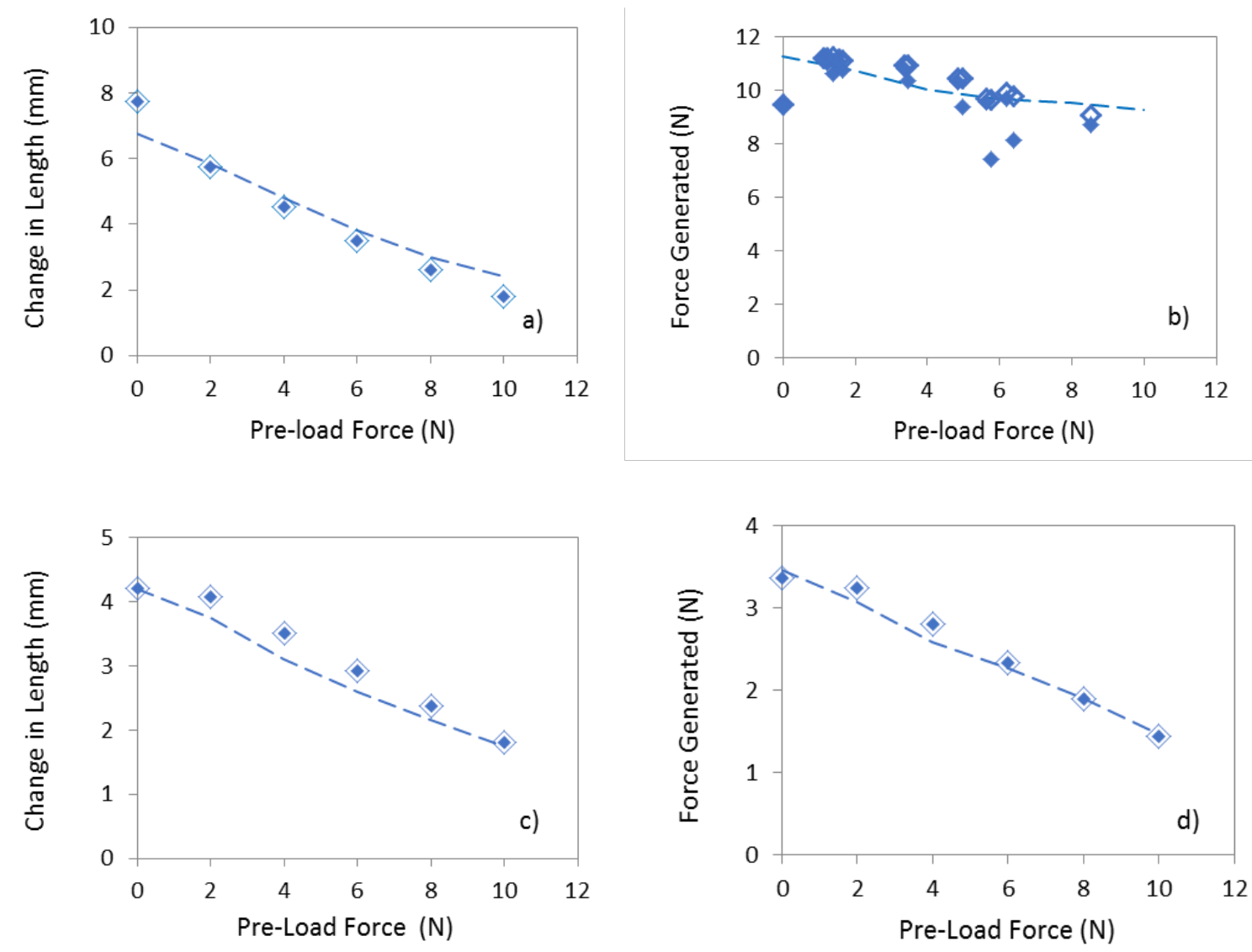

Figure 3 Actuation results for a $71 \mathrm{~mm}$ long braided pneumatic muscle stimulated by pneumatically pressurising from 0 to $100 \mathrm{kPa}$ (solid diamonds) and when depressurising from 100 to $0 \mathrm{kPa}$ (unfilled diamonds). The tests were done under a) isotonic; b) isometric and against a spring in series (c and d). The dashed lines represent the actuator force or stroke predicted by the force-extension curves given in Figure 4.

The loading and unloading curves for the braided muscle were obtained under isobaric conditions at both $0 \mathrm{kPa}$ and $100 \mathrm{kPa}$ internal pressure relative to the ambient pressure (Figure 4). A loadingunloading hysteresis was apparent at both pressures, but was more significant at the higher pressure. The hysteresis introduces a complication to the use of the force-extension curves for the prediction of the actuation, since there are four possible transitions: loading at $0 \mathrm{kPa}$ to loading at 
$100 \mathrm{kPa}$; loading at $0 \mathrm{kPa}$ to unloading at $100 \mathrm{kPa}$; unloading at $0 \mathrm{kPa}$ to loading at $100 \mathrm{kPa}$ and unloading at $0 \mathrm{kPa}$ to unloading at $100 \mathrm{kPa}$. Figure 3 illustrates the situation where the actuation is considered to start at the loading point at $0 \mathrm{kPa}$ ( $\mathrm{A}$ in Fig. 4) and finish on the unloading curve at 100 $\mathrm{kPa}$ for the isotonic (B), isometric (C) and against a spring in series (D). Graphically measuring the change in length and force for each test method and for the same pre-load forces as used in the experiments give the predicted values shown by dashed lines in Figure 3. These estimated actuation parameters were obtained from the transition from the loading curve at $0 \mathrm{kPa}$ to the unloading curve at $100 \mathrm{kPa}$. Excellent agreement is seen between these values estimated from the shift in the forceextension curves due to pressurization and the actual measure isotonic, isometric and against-spring actuation values. Estimates based on transitions to the loading curve at $100 \mathrm{kPa}$ or by using the unloading curve at $0 \mathrm{kPa}$ led to significant over-estimations of the actuation force and stroke, especially in the former case.

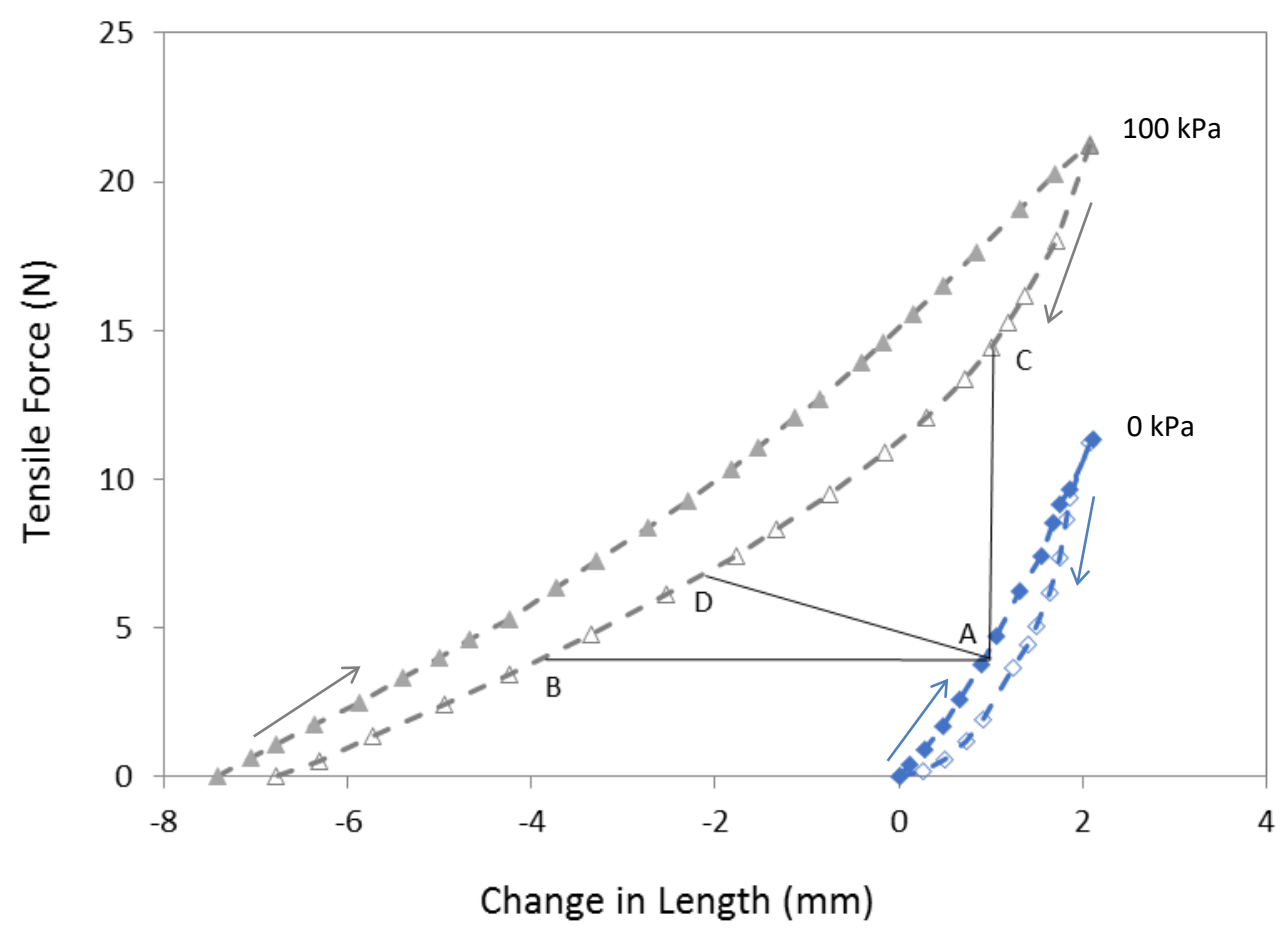

Figure 4 Load-unload curves for braided muscle at atmospheric pressure and after pressurisation to $100 \mathrm{kPa}$ above atmospheric with loading direction indicated by arrows. Solid symbols show loading and unfilled symbols are for unloading. Estimates of the actuation stroke and/or force generated are illustrated by the tie lines A-B (isotonic); A-C (isometric); and A-D (against a spring in series). The latter tie line has a slope corresponding to the external spring stiffness of $0.8 \mathrm{~N} / \mathrm{mm}$.

The same series of actuation tests were repeated for the SMA spring where the sample was fully immersed in a water bath with recirculating water at either $85^{\circ} \mathrm{C}$ or $25^{\circ} \mathrm{C}$. The experimental results are illustrated in Figure 5 and are similar to those previously reported [13]. In contrast to the behaviour of the braided muscles, the SMA coils generate larger forces and larger contraction strokes when higher amounts of pre-stretch were used. The shape memory effect refers to the recovery of deformation introduced into the material below its transition temperature. A significant difference was noted in the force generated and / or length change for all test conditions when comparing the 
first heat / cool cycle with subsequent cycles up to four such cycles. The first heating cycle is often described as a 'training' process and shows smaller forces generated and small strokes than generated in the subsequent heating and cooling cycles. This training effect is evident from the loading and unloading curves of the SMA spring at the starting temperature and before any heating and after heating / cooling. Figure 6 illustrates typical results where the sample is first pre-stretched at $25^{\circ} \mathrm{C}$ to the designated amount, which is $15 \mathrm{~mm}$ in the example shown. Isotonic actuation (Fig. $6 \mathrm{a}$ ) during the first heat results in a small length shortening and a larger lengthening on cooling. Subsequent heat / cool cycles show approximately equivalent shortening and lengthening. Similar behaviour was observed during isometric and series spring actuation tests. In all cases, the final unloaded sample length was increased after the actuation tests when compared to the starting length. The sample could only recover to its initial length by heating without any applied external load.
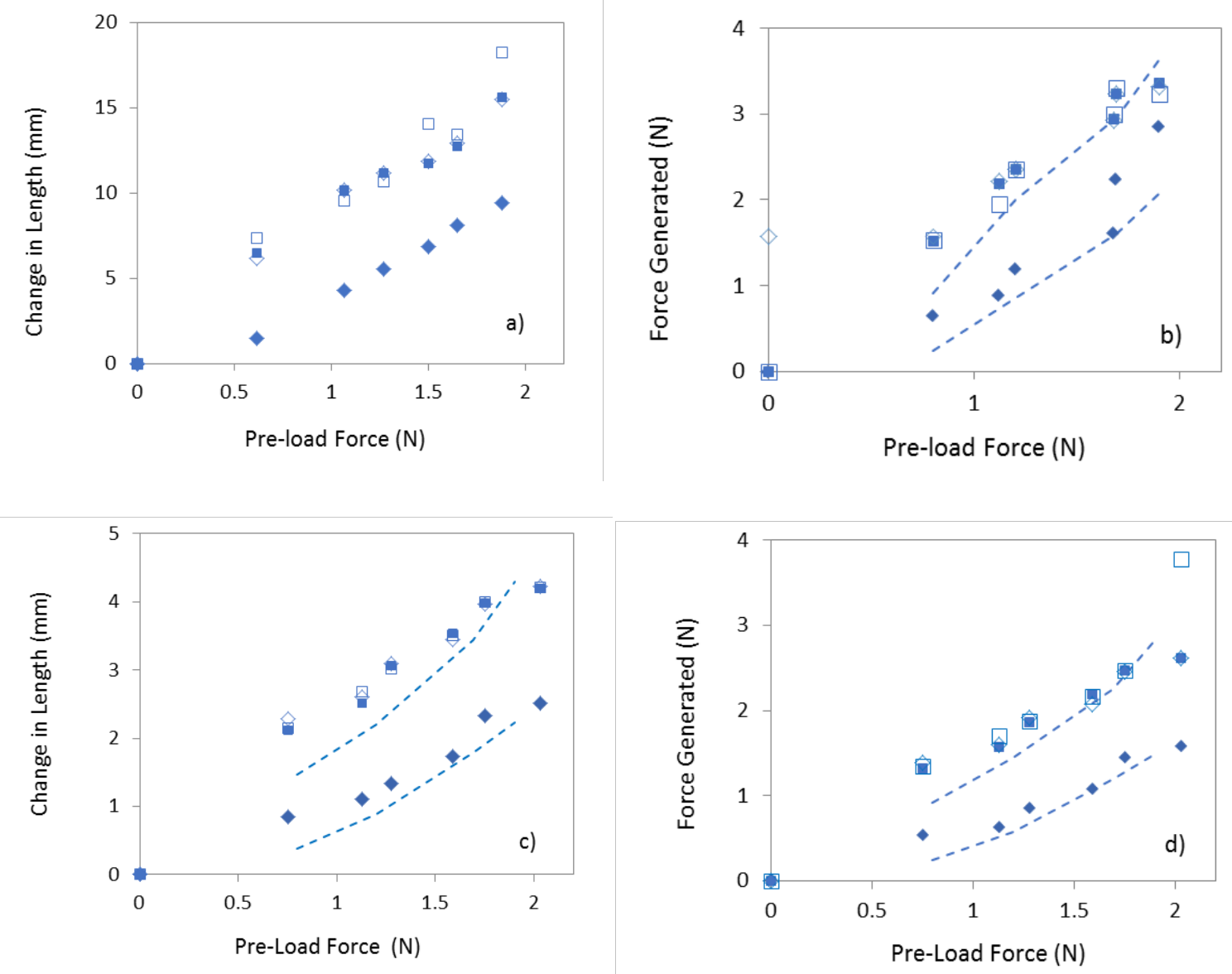

Figure 5 Actuation results for SMA spring stimulated by heating from $25^{\circ} \mathrm{C}$ to $85^{\circ} \mathrm{C}$ and cooling from $85^{\circ} \mathrm{C}$ to $25^{\circ} \mathrm{C}$ and when tested under a) isotonic; b) isometric and against a spring in series (c and d). Test results are shown for the first heating (solid diamonds), the first cooling (unfilled diamonds), the second heating (solid squares) and the second cooling (unfilled squares). The dashed lines give theoretical actuation force and change-in-length estimated by the force-extension curves given in Figure 6. 

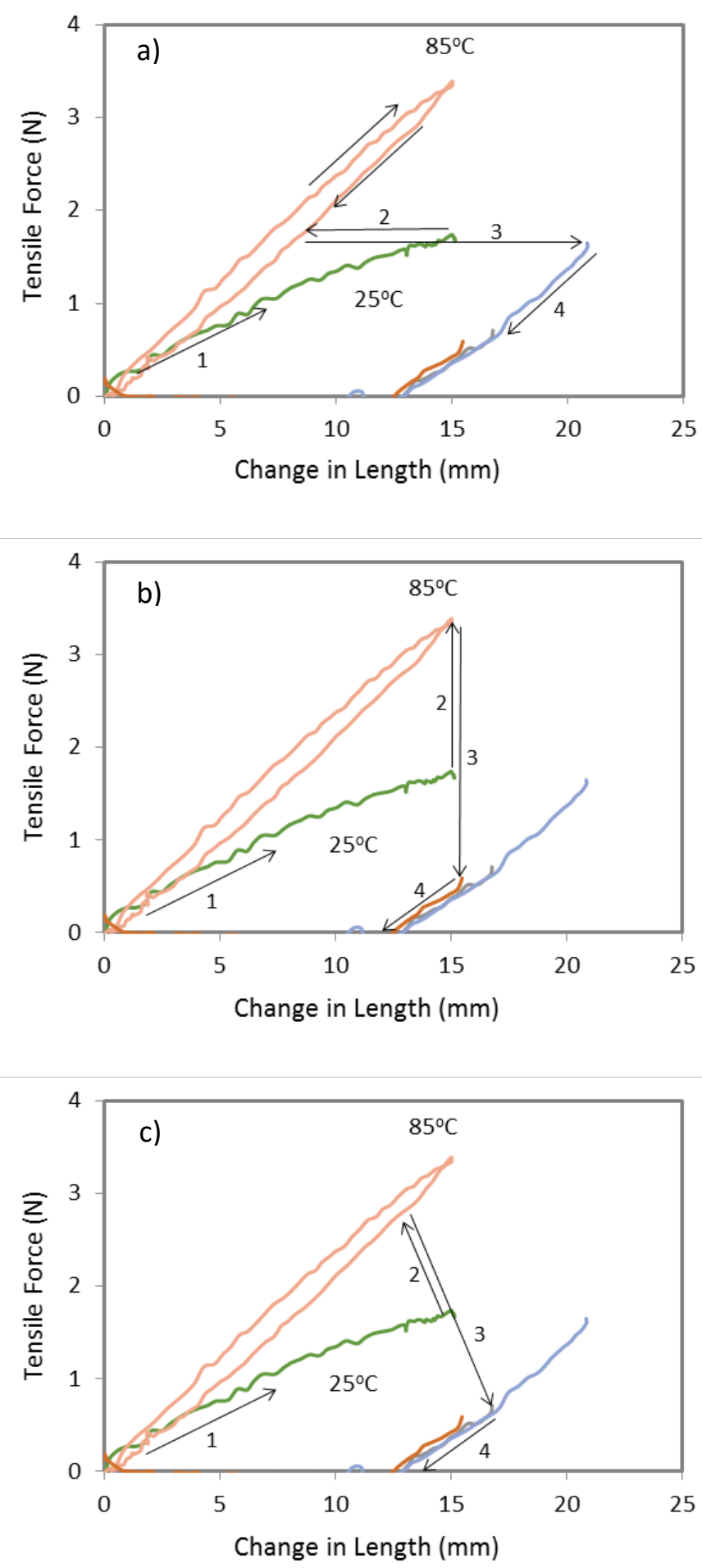

Figure 6 Example actuation results for SMA spring stimulated by heating from $25^{\circ} \mathrm{C}$ to $85^{\circ} \mathrm{C}$ and tested under a) isotonic; b) isometric; and c) against a spring in series. Each testing sequence consisted of (1) pre-stretching to $15 \mathrm{~mm}$ at $25^{\circ} \mathrm{C}$; (2) heating to $85^{\circ} \mathrm{C}$; (3) cooling to $25^{\circ} \mathrm{C}$; and (4) unloading to zero tensile force. Repeated heated and cooling cycles (steps 2 and 3 ) were performed in some tests before step (4). The loading and unloading curves at $85^{\circ} \mathrm{C}$ are shown in each case. 
Importantly, the unloading curves of the SMA spring at $25^{\circ} \mathrm{C}$ was found to be strongly dependent on the sample's thermomechanical history. The unloading curves following isotonic, isometric and series spring actuation tests were coincident for a given initial pre-stretch. However, these unloading curves were right-shifted along the displacement axis when larger pre-stretches were used (Figure 7). It is not possible to predict the magnitude of the length change. Consequently, the prediction of actuation performance using the force-extension curves before and after stimulation becomes problematic with SMAs since there are an infinite number of such curves for the initial temperature state. However, it was possible to predict the actuation behaviour for the isometric and series spring cases by using the experimental results obtained from an isotonic test. As shown as dashed lines in Figure 5, the actuation forces generated in the first heating cycle in an isometric test are reasonably estimated by the vertical distance between the initial loading curve at $25^{\circ} \mathrm{C}$ and the unloading curve at $85^{\circ} \mathrm{C}$, as illustrated by arrow 2 in Figure $6 \mathrm{~b}$ ). Similarly, the forces generated in subsequent heating cycles were adequately estimated by the vertical distance between the $85^{\circ} \mathrm{C}$ unloading curve and the $25^{\circ} \mathrm{C}$ unloading curve following isotonic testing (arrow 3 in Fig. 6b). For actuation tests performed against a spring in series, the first and subsequent heating cycle results were reasonably predicted using the method illustrated by arrows 2 and 3 in Figure $6 c$ ). The slope of the line represented by these arrows corresponds to the stiffness of the series spring $(0.6 \mathrm{~N} / \mathrm{mm})$.

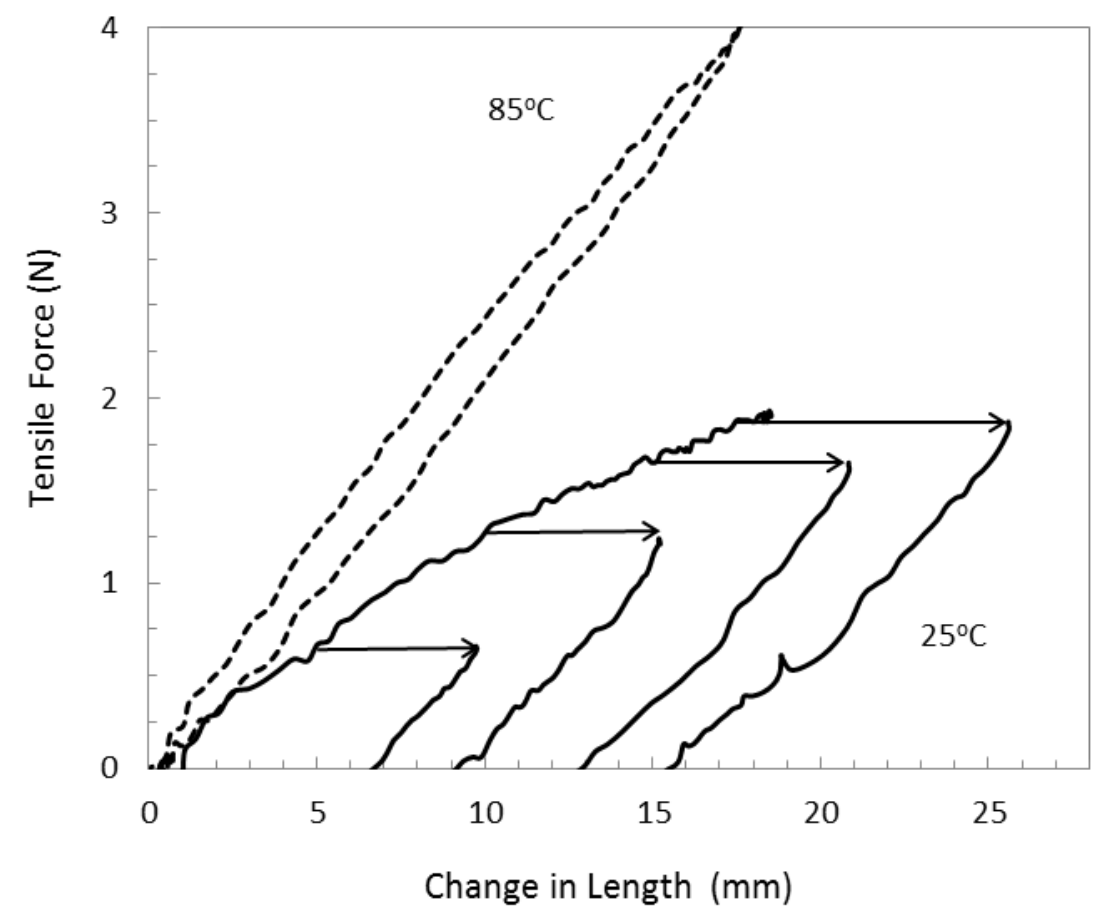

Figure 7 Loading and unloading curves at $85^{\circ} \mathrm{C}$ (dashed lines) and at $25^{\circ} \mathrm{C}$ (solid lines) with unloading following at least one heat / cool cycle at the indicated pre-stretch levels.

Finally, actuation tests were also performed using the twisted and coiled nylon- 6 monofilament. Actuation was initiated by immersion in either hot $\left(85^{\circ} \mathrm{C}\right)$ or cold $\left(25^{\circ} \mathrm{C}\right)$ recirculating water. The experimental results are shown in Figure 8 for isometric, isotonic and against a spring in series. The 
spring constant of the external spring used in the latter tests was $1.87 \mathrm{~N} / \mathrm{mm}$. Highly reversible actuation was obtained even in the first heat/cool cycle, as shown by the similar magnitude of force or length change measured during the heating and cooling phases of the actuation cycle. The forces generated and actuation strokes were found to be independent of the applied pre-load force, at least over the range of pre-loads investigated. Maximum tensile strains were of the order of $9 \%$ and the blocked stress was $\sim 1 \mathrm{MPa}$, based on the external diameter of the unloaded coil.
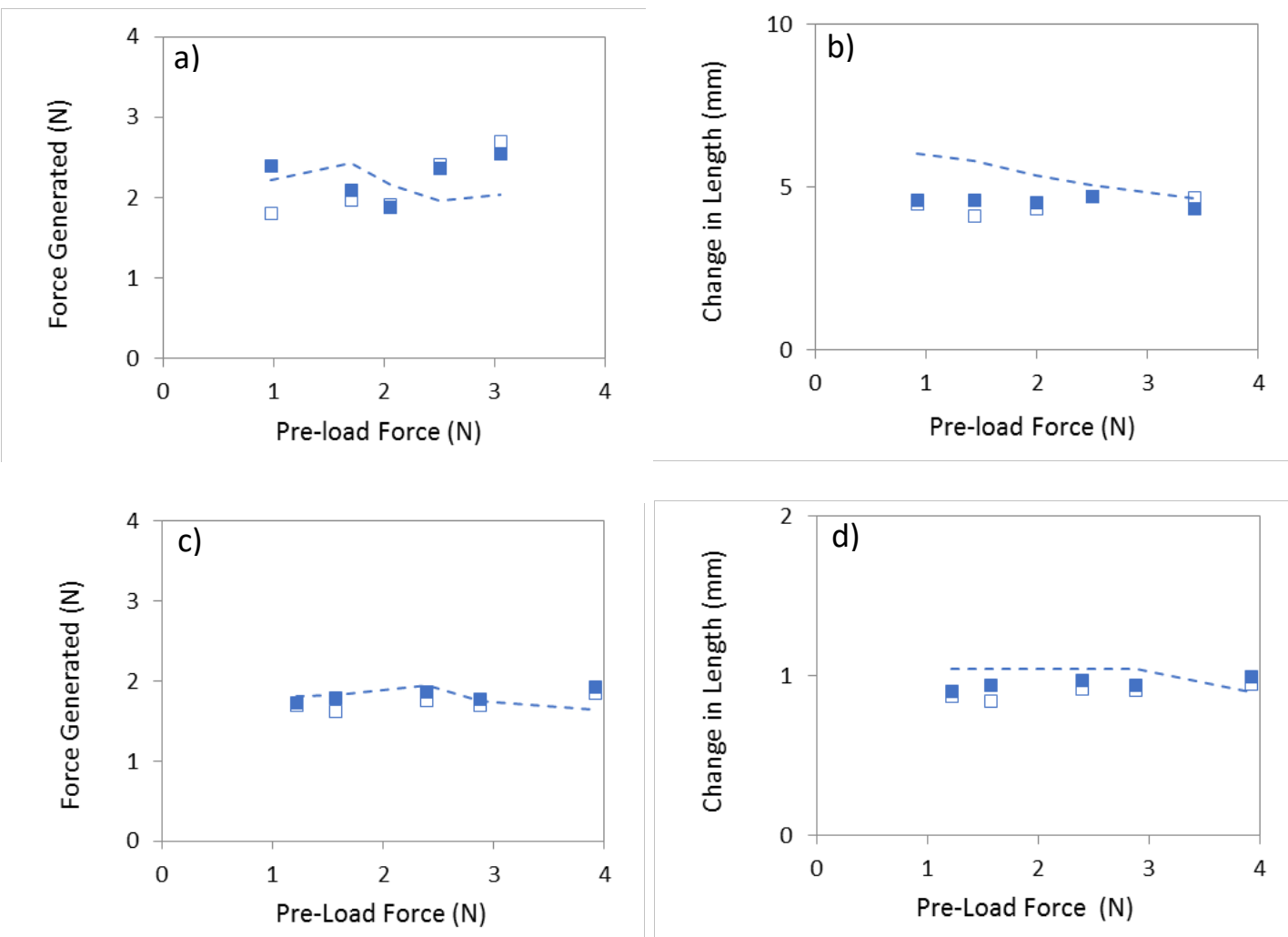

Figure 8 Actuation results for TCP fiber stimulated by heating from $25^{\circ} \mathrm{C}$ to $85^{\circ} \mathrm{C}$ (solid squares) and cooling from $85^{\circ} \mathrm{C}$ to $25^{\circ} \mathrm{C}$ (unfilled squares) and when tested under a) isotonic; b) isometric and against a spring in series ( $c$ and $d$ ). The magnitude of the forces and length changes are shown with heating causing a contraction in length and increase in tensile force. Cooling causes an expansion in length and decrease in tensile force. Dashed lines are calculated values using force-extension curves given in Figure 9.

The force-extension curves obtained at the two temperatures are shown in Figure 9. Good estimates of the experimental actuation results were obtained using the tie-line graphical method from the unloading to the unloading curves at the two temperatures. Example tie-lines for the TCF sample prestretched to $5 \mathrm{~mm}$ are shown in Figure 9 for the isometric (A-B), isotonic (A-C) and series-spring case (A-D). 


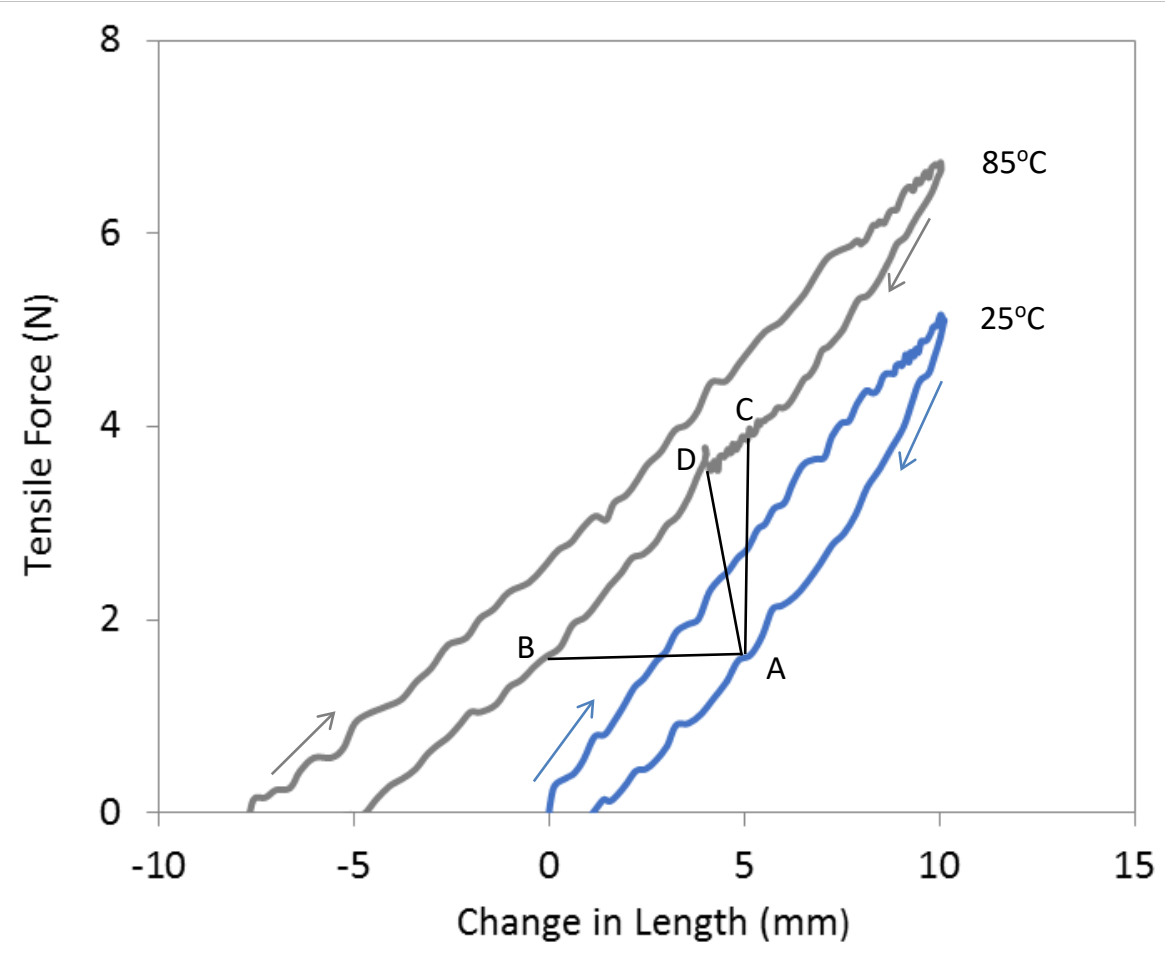

Figure 9 Loading and unloading curves for the TCF sample at $85^{\circ} \mathrm{C}$ and at $25^{\circ} \mathrm{C}$ with loading direction indicated by arrows. Estimates of the actuation stroke and/or force generated after pre-stretching to $5 \mathrm{~mm}$ are illustrated by the tie lines A-B (isotonic); A-C (isometric); and A-D (against a spring in series with a spring constant of $1.87 \mathrm{~N} / \mathrm{mm}$ ).

\section{Conclusions}

A universal actuation test method was developed to estimate the actuation force and stroke generation of three different artificial muscles: a pneumatically operated braided muscle, a thermally operated shape memory alloy (SMA) spring, and a thermally operated twisted and coiled polymer (TCP) fibre. Actuation force and stroke of each artificial muscle were generated at various prestretches following isotonic, isometric, and against an opposing spring testing conditions. Loading/unloading measurements were performed before and after the stimuli input was applied to obtain the force-extension curve of each actuator in the un-activated and activated states, respectively. The transitions between the two curves following each of the three testing modes and at each pre-load were measured to fit the experimental results. It has been found that the forceextension curve method provides very reasonable fits to the isotonic stroke, isometric force, and change in length and force at spring series conditions. The method demonstrates its capability to evaluate the actuation behaviours as to three different artificial muscles with decreased (braided muscle), increased (SMA spring), and un-changed (TCP fibre) stiffness after being activated. Reversible actuation force and stroke were also found for the three tested actuators, as demonstrated by the consistent magnitude of force and change in length. Future work may focus on the challenge that still remains to utilise the method to predict the isotonic displacement of SMA spring which demonstrates a strong dependence on samples' thermomechanical histories. Meanwhile, the hysteresis between loading and unloading curves creates complications, as the 
extent of the hysteresis may affect the measurement of the transitions between the initial and activated force-extension curves. Further work is suggested to better understand the implication of loading and unloading hysteresis.

\section{References}

${ }^{1}$ M. A. McEvoy and N. Correll, "Materials that couple sensing, actuation, computation, and communication" Science, 347, 1261689 (2015). DOI: 10.1126/science.1261689

2 Tissaphern Mirfakhrai, John D.W. Madden, Ray H. Baughman "Polymer artificial muscles" Materials Today, 10, 2007, Pages 30-38

${ }^{3}$ V. Giurgiutiu, C.A. Rogers, A. Rogers, and Z. Chaudhry" Energy based comparison of solidstate induced-strain actuators." J. Intell. Mater. Syst. Struct. 7, 4 (1996).

${ }^{4}$ J. E. Huber, N. A. Fleck and M. F. Ashby "The selection of mechanical actuators based on performance indices" Proc. R. Soc. Lond. A (1997) 453, 2185-2205.

${ }^{5}$ Leonid lonov" "Polymer Actuators" Langmuir 2015, 31, 5015-5024

${ }^{6}$ Aslan Miriyev, Kenneth Stack \& Hod Lipson "Soft material for soft actuators" Nature Communications 8, 596 (2017)

${ }^{7}$ G.M. Spinks "Stretchable artificial muscles from coiled polymer fibers" Journal of Materials Research 31 (2016) 2917-2927.

${ }^{8}$ G.M. Spinks, L. Liu, G.G.Wallace, and D. Zhou "Strain response from polypyrrole actuators under load." Adv. Funct. Mater. 12, 437 (2002).

${ }^{9}$ G.M. Spinks, T.E. Campbell, and G.G. Wallace "Force generation from polypyrrole actuators." Smart Mater. Struct. 14, 406 (2005).

${ }^{10}$ A.T. Asbeck, S.M.M. de Rossi, K.G. Holt, and C.J. Walsh "A biologically inspired soft exosuit for walking assistance." Int. J. Robot. Res. 34, 744 (2015).

${ }^{11}$ Lee Sutton, Hadi Moein, Ali Rafiee, John D.W. Madden, Carlo Menon "Design of an Assistive Wrist Orthosis Using Conductive Nylon Actuators" 6th IEEE RAS/EMBS International Conference on Biomedical Robotics and Biomechatronics (BioRob) June 2629, 2016. UTown, Singapore

12 B. Tondu "Artificial Muscles for Humanoid Robots" in "Humanoid Robots: Human-like Machines" Book edited by: Matthias Hackel ISBN 978-3-902613-07-3, pp. 642, Itech, Vienna, Austria, June 2007

${ }^{13} \mathrm{H}$. Tobushi and K. Tanaka "Deformation of a Shape Memory Alloy Helical Spring" JSME International Journal, 34 (1991), 83-89. 